\title{
EXERCÍCIO FÍSICO COMO FATOR DE PROTEÇÃO PARA A SAÚDE EM SERVIDORES PÚBLICOS
}

\author{
EXERCISE AS PROTECTIVE FACTOR FOR HEALTH OF PUBLIC SERVANTS \\ EJERCICIO COMO FACTOR DE PROTECCIÓN DE LA SALUD EN LOS SERVIDORES PÚBLICOS
}

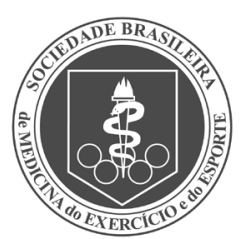

Artigo Original
Maria Lucia de Castro Polisseni (Educadora Física)

Luiz Cláudio Ribeiro' (Demógrafo)

1. Núcleo de Assessoria,

Treinamento e Estudos em Saúde NATES da Universidade Federal de Juiz de Fora, Juiz de Fora, MG, Brasil.

\section{Correspondência:}

Rua do Imperador, 305. Cond. Bosque Imperial, São Pedro. Juiz de Fora, MG, Brasil. 3299791620 marialucia.polisseni@ufjf.edu.br

\section{RESUMO}

Introdução: A atividade física é reconhecida como fator de proteção para a saúde, sendo seus benefícios associados à redução de doenças crônicas e à diminuição do risco de morte prematura por doenças cardiovasculares. Embora a atividade física seja um importante recurso para políticas de promoção de saúde, o baixo nível e a inatividade física são preocupantes em todo o mundo. Em uma sociedade cada vez mais urbanizada e industrializada, exercícios físicos praticados regularmente são importantes recursos para manter o nível de atividade física recomendado, contudo, é preciso identificar as práticas mais adequadas às necessidades preconizadas como efeito protetor do exercício físico para a saúde. Objetivo: Analisar a prevalência de atividade física, fatores sociodemográficos, frequência, modalidades e motivos para a prática de exercício físico em servidores públicos de uma universidade. Métodos: Estudo transversal, amostra aleatória (280 indivíduos) nível de confiança 95\%, erro amostral 5\%. Para avaliar o nível de atividade física foi utilizado o instrumento IPAQ (International Physical Activity Questionnaire), versão curta, na forma de entrevista. Foi aplicado um questionário sociodemográfico e questionado se os indivíduos praticavam exercício físico regularmente, a modalidade e frequência semanal, bem como os motivos para a prática. Os dados foram analisados utilizando-se o software estatístico SPSS - 14.0. Resultados: A prevalência de inativos e insuficientemente ativos foi de 43,9\%. Entre os que fazem exercício físico, $92,5 \%$ o fazem para a saúde $(p<0,005)$, porém $29 \%$ são inativos ou insuficientemente ativos. Das modalidades mais praticadas, ao contrário do esperado, a caminhada não foi significativa. Conclusões: Houve diferenças no nível de atividade física quanto à idade, sexo, estado civil e ocupação, bem como nos motivos, na escolha e diversidade de modalidades praticadas. Além de encorajar e monitorar a atividade física, é preciso criar estratégias institucionais a fim de que se possam obter benefícios de melhor qualidade para a saúde.

Palavras-chave: atividade física, estilo de vida, estudos transversais.

\section{ABSTRACT}

Introduction: Physical activity is recognized as a protective factor for health, and its benefits have been associated to chronic disease reduction and diminished risk of premature death from cardiovascular disease. Although physical activity is an important resource for health promotion policies, low level and physical inactivity are reasons for concern worldwide. In an increasingly urbanized and industrialized society, physical exercises practiced regularly are an important resource to keep the recommended level of physical activity; however, it is necessary to identify the most adequate practices to achieve a protective effect on health. Objective: To analyze the prevalence of physical activity, socio-demographic factors, frequency, modalities and motives for practicing exercises among civil servants from a university. Methods: Cross-sectional study, random sample (280 individuals) confidence level 95\%, 5\% sampling error. The short version of IPAQ (International Physical Activity Questionnaire) instrument was used as an interview. A socio-demographic questionnaire was applied and it was questioned whether subjects practiced exercise regularly, which modality and weekly frequency, as well as the reasons for the practice. Data were analyzed using the statistical package SPSS - 14.0. Results: The prevalence of inactive and insufficiently active subjects was $43.9 \%$. Among those who exercise, $92.5 \%$ did it for health reasons ( $p<0.005)$, but $29 \%$ are inactive or insufficiently active. Of the most widely practiced modality, contrary to expectations, walking was not significant. Conclusions: There were differences in physical activity level according to age, gender, marital status and occupation, as well as the motives, the choice and diversity of modalities practiced. In addition to encouraging and monitoring the physical activity, it is necessary to create institutional strategies in order to promote better quality benefits for health.

Keywords: motor activity, life style, cross-sectional studies.

\section{RESUMEN}

Introducción: La actividad física es reconocida como un factor de protección para la salud, siendo sus beneficios asociados a la reducción de enfermedades crónicas y a la disminución del riesgo de muerte prematura por enfermedades cardiovasculares. Aunque la actividad física es un recurso importante para las politicas de promoción de salud, el bajo nivel y la inactividad física son preocupantes en todo el mundo. En una sociedad cada vez más urbanizada e industrializada, los ejercicios físicos practicados regularmente son instrumentos importantes para mantener el nivel recomendado de 
actividad física, sin embargo, es necesario identificar las prácticas más adecuadas a las necesidades propuestas como efecto protector del ejercicio físico para la salud. Objetivo: Analizar la prevalencia de actividad física, factores sociodemográficos, frecuencia, modalidades y motivos para la práctica de ejercicios físicos en servidores públicos de una universidad. Métodos: Estudio transversal, muestra aleatoria (280), nivel de confianza 95\%, error de muestreo 5\%. Para evaluar el nivel de actividad física fue usado el instrumento IPAQ (International Physical Activity Questionnaire) versión corta en la forma de entrevista. Fue aplicado un cuestionario sociodemográfico y se cuestionó si los individuos practicaban ejercicio físico con regularidad, la modalidad y frecuencia semanal, así como los motivos para la práctica. Los datos fueron analizados utilizando el software estadístico SPSS - 14.0. Resultados: La prevalencia de inactivos e insuficientemente activos fue de 43,9\%. Entre los que hacen ejercicio, 92,5\% lo hacen para la salud ( $p<0,005$ ), pero $29 \%$ son inactivos o insuficientemente activos. De las modalidades más practicadas, al contrario de lo esperado, la caminata no fue significativa. Conclusiones: Hubo diferencias en el nivel de actividad física cuanto a la edad, el sexo, el estado civil y la ocupación, bien como en los motivos, la elección y la diversidad de modalidades practicadas. Además de fomentar y supervisar la actividad física es necesario crear estrategias institucionales para que se puedan obtener beneficios de mejor calidad para la salud.

Palabras clave: actividad física, estilo de vida, estudios transversales.

\section{INTRODUÇÃO}

A atividade física tem sido associada como fator de proteção para a saúde desde a década de 1950¹. Desde então, estudos têm associado seus benefícios à redução de doenças crônicas² à diminuição do peso em adultos praticantes de atividade aeróbica ao longo da vida ${ }^{3}$ ou ainda à diminuição do risco de morte prematura por doenças cardiovasculares ${ }^{4}$.

Constatações como essas têm levado ao reconhecimento da prática de atividade física como importante recurso para políticas de promoção de saúde ${ }^{5}$. No entanto, o baixo nível de atividade física e a inatividade física são preocupantes em todo o mundo ${ }^{6,7}$.

As prevalências de inatividade física verificadas em pessoas adultas de diversos países, em 2008, foram acima de 10\%. No Brasil, havia $26,1 \%$ de inativos. Observou-se similaridade no nível de atividade física e fatores associados em pessoas até 49 anos para ambos os sexos ${ }^{6}$. Em 2011, em média, uma em cada cinco pessoas no mundo foram consideradas fisicamente inativas, sendo a inatividade física mais prevalente em países urbanizados, entre mulheres e idosos? ${ }^{7}$.

A atividade física é identificada como qualquer movimento corporal produzido pelo sistema musculoesquelético que resulta em gasto energético ${ }^{8}$, podendo ser investigada sob as óticas do lazer, isto é, aquela realizada no tempo livre, incluindo esportes, caminhadas e exercícios fiscos, ou sob a ótica global, que inclui, além das primeiras, as atividades domésticas, de deslocamento e ocupacionais ${ }^{9}$. 0 American College of Sports Medicine ${ }^{10}$ recomenda, para promoção de saúde e prevenção de doenças em adultos com idades entre 18 e 64 anos, fazer pelo menos 150 minutos de atividade física aeróbica, de intensidade moderada, por semana, ou pelo menos 75 minutos de atividade física aeróbica, de intensidade vigorosa, por semana, ou, ainda, a combinação de ambos, podendo ser realizadas em frações de pelo menos 10 minutos de duração e distribuídos durante a semana. Recomenda-se também realizar atividades de força muscular, moderadas ou vigorosas, duas ou mais vezes por semana.

Numa sociedade cada vez mais urbanizada e industrializada, os exercícios físicos praticados regularmente são importantes recursos para manter o nível de atividade física recomendado. No entanto, estudos têm verificado que diversos fatores são associados negativamente à sua prática. Estudo feito em Andaluzia, na Espanha, verificou que grupos menos favorecidos e com menor grau de instrução são menos prováveis de se engajarem em exercícios físicos ${ }^{11}$. Outros fatores, como o casamento para homens, a alocação do tempo e muIheres com crianças dependentes tiveram impacto negativo para a frequência à prática de exercício físico entre trabalhadores na Austrália ${ }^{12}$.
Os efeitos benéficos dos exercícios físicos estão bem descritos na literatura. Porém, em se tratando de escolhas e motivos para a prática de exercícios, é preciso identificar as práticas mais adequadas às necessidades preconizadas como efeito protetor do exercício físico para a saúde ${ }^{13}$.

Nas últimas décadas, modelos e teorias comportamentais têm sido utilizados na orientação de programas de promoção de atividade físi$\mathrm{ca}^{14}$. Os efeitos desse tipo de intervenção têm sido constatados como pequenos e temporários em níveis comunitários ${ }^{14}$, visto que apoiam suas estratégias em mudanças comportamentais individuais como meio de redução do risco epidemiológico, independentemente dos condicionantes sociais, econômicos e culturais ${ }^{15}$.

A posição da sociedade de medicina do esporte é de que a atividade física deve ser incentivada e estimulada para a preservação da saúde, por meio de iniciativas tanto do poder público quanto do privado $^{16}$. Portanto, compreender escolhas individuais no contexto social em que a pessoa vive como forma de conhecimento, além do nível de atividade física e fatores associados, dos motivos para o comportamento motor dos indivíduos pode contribuir para traçar estratégias mais adequadas de promoção da saúde, visando atingir coletivamente grupos específicos na busca da superação do caráter efêmero dos resultados obtidos pelos programas atuais.

Este estudo teve por objetivo verificar a prevalência de atividade física e fatores associados em servidores federais de uma universidade pública do sudeste do Brasil, bem como avaliar a frequência, as modalidades e os motivos para a prática de exercícios físicos regulares.

\section{MATERIAL E MÉTODO}

Estudo transversal realizado em população específica de docentes e técnicos administrativos em educação da Universidade Federal de Juiz de Fora, MG, Brasil. Foi selecionada uma amostra aleatória cujo tamanho foi calculado considerando-se a prevalência de inatividade física de 26,1\% nível de confiança de 95\%, erro amostral de 5\% e tamanho populacional de 2375. A amostra final foi constituída de 280 participantes. Como critério de exclusão adotou-se a incapacidade física de realizar exercício físico.

Para a obtenção dos dados foram utilizados o Questionário Internacional de Nível de Atividade Física (IPAQ) versão curta e um Questionário sociodemográfico. Apesar do IPAQ ser autoaplicável, a coleta foi realizada em forma de entrevista, visto que este procedimento tem proporcionado melhor qualidade dos dados ${ }^{17}$. Este instrumento, que contempla as facetas de atividades domésticas, de deslocamento, ocupacionais e de lazer foi desenvolvido, validado e adaptado em diversos países ${ }^{18,19}$. 
O IPAQ versão curta afere o nível de atividade física global realizada no trabalho, no lazer, como meio de transporte e em atividades domésticas. Além disso, é questionado o tempo em que o indivíduo permanece sentado em um dia típico de semana e em um dia típico de fim de semana. No questionário sociodemográfico, além das questões de identificação dos sujeitos, foi questionado se eles praticavam exercício físico ao menos uma vez por semana, as modalidades (considerando o gasto energético ${ }^{20}$ e frequência, bem como os motivos para a prática.

Os dados foram analisados utilizando-se o software estatístico SPSS 14.0 (Statistical Package for Social Sciences).

Os procedimentos adotados para a realização desta pesquisa estão de acordo com as diretrizes e normas da resolução no 196 do Conselho Nacional de Saúde, de 10 de outubro de 1996, para pesquisas que envolvem seres humanos, tendo sido aprovado pelo Comitê de Ética da Universidade Federal de Juiz de Fora, Juiz de Fora, MG, Brasil (HU/ UFJF) parecer $n^{0}$ CCAAE - 0030.0.420.000-11.

\section{RESULTADOS}

Foram analisados 280 indivíduos. A média de idade foi de 47,5 anos (desvio padrão=9,7; mediana $=49$ anos) sendo $57,5 \%$ do sexo masculino. A prevalência de sujeitos inativos foi de 10,0\%; insuficientemente ativos 33,9\%; ativos $45,7 \%$ e muito ativos $10,4 \%$. Dentre os classificados como ativos em nível suficiente, $77,7 \%$ declararam praticar exercício físico regularmente. A tabela 1 apresenta a distribuição do Nível de Atividade Física segundo variáveis sociodemográficas.

Na amostra geral, os técnicos administrativos em educação (61\%) foram significantemente mais ativos do que os docentes $(p=0,042)$.

Apesar de não haver diferenças significativas na maioria dos fatores pesquisados para a amostra geral, foi feita estratificação por idade, visto que este é um fator relevante para o padrão de atividade física dos indivíduos.

Observando apenas as pessoas até 49 anos 62,2\% das mulheres foram classificadas como ativas ou muito ativas, sendo que $49,3 \%$ dos homens ficaram nas mesmas categorias. Os indivíduos solteiros, viúvos, separados ou divorciados, nesta faixa etária, foram mais ativos do que os casados ou com união estável $(67,9 \%$ e 48,4\%, respectivamente, $p=0,015)$.

Tabela 1. Nível de atividade física segundo variáveis sociodemográficas.

\begin{tabular}{|c|c|c|c|c|c|}
\hline \multirow[t]{2}{*}{ Fatores } & \multicolumn{2}{|c|}{$\begin{array}{c}\text { Inativos ou } \\
\text { insuficientemente } \\
\text { ativos }\end{array}$} & \multicolumn{2}{|c|}{$\begin{array}{l}\text { Ativos ou muito } \\
\text { ativos }\end{array}$} & \multirow[t]{2}{*}{$p$} \\
\hline & $\mathrm{N}$ & $(\%)$ & $\mathrm{N}$ & (\%) & \\
\hline Sexo & & & & & 0,522 \\
\hline Masculino & 71 & 44,1 & 90 & 55,9 & \\
\hline Feminino & 52 & 43,7 & 67 & 56,3 & \\
\hline Idade & & & & & 0,496 \\
\hline Até 49 anos & 66 & 44,3 & 83 & 55,7 & \\
\hline 50 anos ou mais & 57 & 43,5 & 74 & 56,5 & \\
\hline Cor da Pele & & & & & 0,220 \\
\hline Branca & 92 & 45,5 & 110 & 54,5 & \\
\hline Não branca & 30 & 39,5 & 46 & 60,5 & \\
\hline Estado Civil & & & & & 0,343 \\
\hline $\begin{array}{l}\text { Casado ou com } \\
\text { união estável }\end{array}$ & 79 & 45,1 & 96 & 54,9 & \\
\hline $\begin{array}{c}\text { Solteiro, viúvo, separado } \\
\text { ou divorciado }\end{array}$ & 44 & 41,9 & 61 & 58,1 & \\
\hline Escolaridade & & & & & 0,130 \\
\hline Até $2^{\circ}$ grau & 12 & 32,4 & 25 & 67,6 & \\
\hline $3^{0}$ grau ou pós-graduado & 111 & 45,6 & 132 & 54,4 & \\
\hline Ocupação & & & & & 0,042 \\
\hline Docente & 63 & 50 & 63 & 50 & \\
\hline $\begin{array}{l}\text { Técnico Administrativo } \\
\text { em Educação }\end{array}$ & 60 & 39 & 94 & 61 & \\
\hline
\end{tabular}

Na faixa etária acima de 49 anos, houve diminuição na frequência de ativos ou muito ativos para solteiros, viúvos, separados ou divorciados (de 67,9\% para 46,9\%) e aumento para indivíduos casados (de 48,4\% para 62,2\%). Quanto ao sexo, nesta faixa etária, houve diminuição de ativos ou muito ativos para mulheres (de 62,2\% para 46,7\%) e aumento para homens (de 49,3\% para 61,6\%). As prevalências de atividade física por idade apresentadas na tabela 2.

Quanto à declaração de prática regular de exercício físico, 61,4\% dos indivíduos declararam praticar duas ou mais vezes por semana. No entanto, 29\% destes foram classificados como inativos ou insuficientemente ativos. A chance do indivíduo que pratica exercício físico regularmente duas vezes por semana ser classificado como inativo ou insuficientemente ativo é de 2,44 vezes maior do que aquele que pratica três vezes por semana. As modalidades mais praticadas e os motivos para a prática estão dispostos na tabela 3.

Tabela 2. Prevalência de atividade física por idade.

\begin{tabular}{|c|c|c|c|c|c|c|c|c|c|c|}
\hline \multirow{3}{*}{ Fatores } & \multicolumn{4}{|c|}{ Indivíduos até 49 anos } & \multirow{3}{*}{$p$} & \multicolumn{4}{|c|}{$\begin{array}{l}\text { Indivíduos com } \\
50 \text { anos ou mais }\end{array}$} & \multirow{3}{*}{$P$} \\
\hline & \multicolumn{2}{|c|}{$\begin{array}{c}\text { Inativos ou } \\
\text { insuficien- } \\
\text { temente } \\
\text { ativos }\end{array}$} & \multicolumn{2}{|c|}{$\begin{array}{c}\text { Ativos ou } \\
\text { muito ativos }\end{array}$} & & \multicolumn{2}{|c|}{$\begin{array}{c}\text { Inativos ou } \\
\text { insuficien- } \\
\text { temente } \\
\text { ativos }\end{array}$} & \multicolumn{2}{|c|}{$\begin{array}{c}\text { Ativos ou } \\
\text { muito ativos }\end{array}$} & \\
\hline & $\mathrm{N}$ & $(\%)$ & $\mathrm{n}$ & $(\%)$ & & $\mathrm{n}$ & $(\%)$ & $\mathrm{N}$ & $(\%)$ & \\
\hline Sexo & & & & & 0,079 & & & & & 0,073 \\
\hline Masculino & 38 & 50,7 & 37 & 49,3 & & 33 & 38,4 & 53 & 61,6 & \\
\hline Feminino & 28 & 37,3 & 46 & 62,2 & & 24 & 53,3 & 21 & 46,7 & \\
\hline Cor da Pele & & & & & 0,499 & & & & & 0,104 \\
\hline Branca & 50 & 43,9 & 64 & 56,1 & & 42 & 47,7 & 46 & 52,3 & \\
\hline Não branca & 16 & 45,7 & 19 & 54,3 & & 14 & 34,1 & 27 & 65,9 & \\
\hline Estado Civil & & & & & 0,015 & & & & & 0,064 \\
\hline $\begin{array}{c}\text { Casado ou com } \\
\text { união estável }\end{array}$ & 48 & 51,6 & 45 & 48,4 & & 31 & 37,8 & 51 & 62,2 & \\
\hline $\begin{array}{c}\text { Solteiro, } \\
\text { viúvo,separado } \\
\text { ou divorciado } \\
\end{array}$ & 18 & 32,1 & 38 & 67,9 & & 26 & 53,1 & 23 & 46,9 & \\
\hline Escolaridade & & & & & 0,259 & & & & & 0,185 \\
\hline Até $2^{0}$ grau & 2 & 25,0 & 6 & 75,0 & & 10 & 34,5 & 19 & 65,5 & \\
\hline $\begin{array}{c}3^{0} \text { grau ou } \\
\text { pós-graduado }\end{array}$ & 64 & 45,4 & 77 & 54,6 & & 47 & 46,1 & 65 & 63,7 & \\
\hline Ocupação & & & & & 0,073 & & & & & 0,211 \\
\hline Docente & 35 & 51,5 & 33 & 48,5 & & 28 & 48,3 & 30 & 51,7 & \\
\hline $\begin{array}{c}\text { Técnico } \\
\text { Administrativo } \\
\text { em Educação }\end{array}$ & 31 & 38,3 & 50 & 61,7 & & 29 & 39,7 & 44 & 60,3 & \\
\hline
\end{tabular}

Tabela 3. Modalidades de prática de exercício físico regular e motivos para a prática / Nível de atividade física ( $\mathrm{n}=172$ ).

\begin{tabular}{c|c|c|c|c|c}
\hline \multirow{2}{*}{ Modalidades/motivos } & \multicolumn{2}{|c|}{$\begin{array}{c}\text { Inativos ou } \\
\text { insuficientemente ativos }\end{array}$} & $\begin{array}{c}\text { Ativos ou muito } \\
\text { ativos }\end{array}$ & \multirow{2}{*}{$\mathbf{p}$} \\
\cline { 2 - 5 } & $\mathbf{n}$ & $\mathbf{( \% )}$ & $\mathbf{n}$ & $\mathbf{( \% )}$ & \\
\hline Modalidades & & & & & \\
\hline Musculação & 13 & 22,4 & 45 & 77,6 & $<0,001$ \\
\hline Corrida & 7 & 15,6 & 38 & 84,4 & $<0,001$ \\
\hline Caminhada & 11 & 35,5 & 20 & 64,5 & 0,106 \\
\hline Pilates & 9 & 30 & 21 & 70 & 0,028 \\
\hline $\begin{array}{c}\text { Motivos para prática de } \\
\text { exercício físico }\end{array}$ & & & & & \\
\hline Para a saúde & 49 & 30,8 & 110 & 69,2 & $<0,001$ \\
\hline Por estética & 18 & 28,6 & 45 & 71,4 & $<0,001$ \\
\hline Por prazer em exercitar-se & 23 & 23,5 & 75 & 76,5 & $<0,001$ \\
\hline Para estar com amigos & 8 & 47,1 & 9 & 52,9 & 0,808 \\
\hline Para fazer novos amigos & 4 & 33,3 & 8 & 66,7 & 0,248 \\
\hline
\end{tabular}


Foram declaradas 26 diferentes modalidades de prática de exercício físico. Levando em consideração todos os indivíduos, as modalidades mais praticadas foram: musculação $(20,7 \%)$ e a corrida $(16,1 \%)$, caminhada $(11,0 \%)$ e pilates $(10,7 \%)$. Dessas modalidades, apenas a caminhada não se associou ao nível de atividade física de modo significativo.

Dentre os indivíduos que declararam fazer exercício físico regular, 92,5\% disserem fazê-lo para a saúde. O motivo saúde foi o mais prevalente entre os motivos declarados $(86,9 \%)$, seguido do prazer em exercitar-se $(53,6 \%)$ e da estética $(34,4 \%)$. Foram verificadas diferenças significativas $(p<0,001)$ em relação à prática de exercício físico por esses três motivos. Os motivos relativos à interação social (estar com amigos e fazer novos amigos) não apresentaram significância.

Também foi verificado o tempo de permanência sentado. Durante um dia típico de semana a média foi de 5h42min (Desvio Padrão de 2h59min) e durante um dia típico de fim de semana de 4h39min (Desvio Padrão de 3h02min). Para os indivíduos até 49 anos, o tempo de permanência sentado durante a semana associou-se ao nível de atividade física $(\mathrm{p}=0,024)$. Para o sexo masculino, associou-se ao tempo de permanência sentado durante um dia típico de fim de semana $(p=0,011)$.

Quanto à ocupação, os Técnicos Administrativos em Educação apresentaram menor tempo de permanência sentado durante um dia típico de semana, ou seja, abaixo da média de 5h42min (61,3\%; $p=0,022)$ e, da mesma forma, menor tempo de permanência sentado durante um dia típico de fim de semana, ou seja, abaixo da média de 4h39min (61,3\%; $p=0,011)$.

\section{DISCUSSÃO}

A prevalência de sujeitos inativos foi de $10 \%$. Considerando a margem de erro de nosso estudo de 5\%, esses valores são similares aos encontrados em adultos, nas capitais brasileiras, através do inquérito telefônico ${ }^{21}$ que registrou prevalência de 14,9\%, havendo variação de 11,4\% em Florianópolis até 18,4\% no Recife.

Embora a inatividade física venha caindo no Brasi|21, estudos têm procurado verificá-la em conjunto com o nível de atividade física insuficiente, considerado incapaz de garantir os benefícios esperados para a saúde. Estudos realizados com população adulta registraram $77,7 \%$ de inativos ou insuficientemente ativos no lazer no Estado de São Paulo ${ }^{22}$ e 52,7\% em Florianópolis ${ }^{23}$. Em funcionários de uma universidade do Estado da Bahia registrou-se 49,4\% de funcionários insuficientemente ativos ${ }^{24}$. Em nosso estudo foi verificado 56,43\% de inativos ou insuficientemente ativos no lazer (entre os praticantes de exercícios físicos regulares) e $43,9 \%$ no geral, sendo que entre docentes a prevalência foi de $50 \%$.

As frequências observadas para homens e mulheres foram similares. O mesmo não se verificou quando foram observadas apenas as pessoas acima de 49 anos, confirmando uma tendência mundial de diminuição do nível de atividade física para mulheres mais velhas ${ }^{6,7}$.

Foram verificadas diferenças significativas quanto à ocupação. Os técnicos administrativos em educação foram classificados como mais ativos e que permanecem menor tempo sentado tanto durante um dia típico de semana quanto durante um dia típico de fim de semana. Este resultado pode estar relacionado ao tipo de trabalho realizado, visto que em relação à prática de exercícios físicos as prevalências são similares. Neste sentido, há de se observar, também, o contexto atual do trabalho docente de instituições públicas de ensino superior, no Brasil, no que se refere à produção do conhecimento. Há sobrecarga de trabalho pelas exigências próprias do fazer acadêmico (ministrar aulas, orientar alunos, corrigir trabalhos, pesquisar, participar de reuniões deliberativas, dentre outras) e que são consideradas invisíveis, e há metas produtivistas palpáveis (produção científica) que levam os docentes a aumentarem suas cargas de trabalho com extensão ao nível doméstico ${ }^{25}$.

A prática de atividade física no lazer foi registrada em separado do contexto global pela prática e frequência de exercícios físicos declarados $(61,4 \%)$, sendo encontrados neste grupo 29\% de inativos ou insuficientemente ativos. Esta diferença pode ser explicada por uma possível superestimação das respostas, pois, mesmo tendo sido mais observadas no IPAQ no âmbito doméstico e laboral23, as pessoas tendem a valorizar a resposta supostamente positiva. Ao levar em consideração esta característica do IPAQ, pode-se dizer que estes percentuais poderiam ser ainda maiores. Estes achados podem estar também relacionados às características das modalidades praticadas em relação à frequência, classificação de dispêndio energético ${ }^{23}$ ou percepção do próprio esforço. Atividades físicas a nível insuficiente podem auxiliar na diminuição dos danos causados pela inatividade física ${ }^{4}$, porém, escolha da modalidade mais adequada tem sido apontada como uma questão importante para a obtenção dos benefícios esperados ${ }^{13}$.

Dentre as modalidades, houve um número significantemente maior de indivíduos classificados como ativos entre os que praticam musculação, corrida e pilates. Ao observar as características de prática dessas atividades, destaca-se que a musculação, classificada como atividade moderada, em geral é praticada com alta frequência semanal; a corrida, por ser uma atividade vigorosa, mesmo com tempo relativamente menor de prática semanal, poderá cobrir as necessidades cardiovasculares preconizadas e o pilates, embora seja uma prática classificada como suave ou moderada, teve sua frequência associada a outras modalidades em 100\%. Diferentemente do esperado, o número de pessoas que praticam a modalidade caminhada, preconizada como atividade de escolha para a manutenção do nível de atividade física, devido ao custo inexistente e alcance de todos, não foi significantemente diferente entre os que foram classificados como ativos ou como inativos (ou insuficientemente ativos).

Outro achado relevante em nosso estudo foi que dos indivíduos declararam fazer exercício físico $92,5 \%$ o fazem para a saúde, porém $29 \%$ deles não estão cobertos por suas práticas. Isso aponta para a necessidade de reflexão sobre a prática adequada do exercício ${ }^{13}$ e o papel do profissional de Educação Física e das instituições na promoção de saúde e atividade física. Em revisão sistemática ${ }^{13}$, de 2013, sobre as intervenções em atividade física na América Latina, foram classificadas como promissoras as sessões de atividade física em ambientes comunitários, os programas integrados, o acesso a locais para a prática de atividade física combinado com atividades informativas. Esse tipo de intervenção em ambiente institucional tira as responsabilidades exclusivamente individuais quanto ao cuidado de si na prevenção de doenças e coloca a cargo de profissionais o monitoramento, acompanhamento e orientação das práticas.

Quanto aos motivos para a prática de exercício físico, observou-se que as motivações individuais (saúde, estética e prazer em exercitar-se) tiveram maior frequência entre o grupo de ativos ou muito ativos do que entre o grupo dos inativos ou insuficientemente ativos, e foram significativas. O mesmo não ocorreu para as motivações de interação social (estar com amigos e fazer novos amigos). Em revisão sistemática sobre determinantes do nível de atividade física, constatou-se em pesquisas sobre variantes genéticas, dentre elas, receptores dopaminérgicos, relacionados à sensação de prazer, que eles estão associados, de forma consistente, à atividade física ${ }^{26}$. 


\section{CONCLUSÕES}

Os resultados encontrados mostraram que apesar da grande maioria dos indivíduos declararem fazer exercício físico para a saúde, muitos deles não o fazem em volume adequado para se beneficiarem da atividade física como fator associado à proteção para a saúde.

Em nível coletivo, além de encorajar e monitorar a atividade física são necessárias estratégias institucionais e orientação profissional a fim de que se possam obter benefícios maiores e de melhor qualidade para a saúde pela prática de exercícios físicos.

Todos os autores declararam não haver qualquer potencial conflito de interesses referente a este artigo.

\section{REFERÊNCIAS}

1. Shiroma EJ, Lee IM. Physical activity and cardiovascular health: lessons learned from epidemiological studies across age, gender, and race/ethnicity. Circulation. 2010;122(7):743-52.

2. Harati H, Hadaegh F, Momenan AA, Ghanei L, Bozorgmanesh MR, Ghanbarian A, et al. Reduction in incidence of type 2 diabetes by lifestyle intervention in a middle eastern community. Am J Prev Med. 2010;38(6):628-636.e1

3. Donnelly JE, Blair SN, Jakicic JM, Manore MM, Rankin JW, Smith BK; American College of Sports Medicine American College of Sports Medicine Position Stand. Appropriate physical activity intervention strategies for weight loss and prevention of weight regain for adults. Med Sci Sports Exerc. 2009;41(2):459-71.

4. Zhao G, Li C, Ford ES, Fulton JE, Carlson SA, Okoro CA, et al. Leisure-time aerobic physical activity, muscle-strengthening activity and mortality risks among US adults: the NHANES linked mortality study. Br J Sports Med. 2014;48(3):244-9.

5. Brasil. Ministério da Saúde. Secretaria de Vigilância em Saúde. Política nacional de promoção da saúde / Ministério da Saúde, Secretaria de Atenção à Saúde. - Brasília: Ministério da Saúde. Brasília: Ministério da Saúde; 2006.

6. Guthold R, Ono T, Strong KL, Chatterji S, Morabia A. Worldwide variability in physical inactivity a 51-country survey. Am J Prev Med. 2008;34(6):486-94.

7. Dumith SC, Hallal PC, Reis RS, Kohl HW 3rd. Worldwide prevalence of physical inactivity and its association with human development index in 76 countries. Prev Med. 2011;53(1-2):24-8.

8. Caspersen CJ, Powell KE, Christenson GM. Physical activity, exercise, and physical fitness: definitions and distinctions for health-related research. Public Health Rep. 1985;100(2):126-31.

9. Hagströmer M, Oja P, Sjöström M. The International Physical Activity Questionnaire (IPAQ): a study of concurrent and construct validity. Public Health Nutr. 2006;9(6):755-62.

10. Pate RR, Pratt M, Blair SN, Haskell WL, Macera CA, Bouchard C, et al. Physical activity and public health. A recommendation from the Centers for Disease Control and Prevention and the American College of Sports Medicine. JAMA. 1995;273(5):402-7.

11. Bolívar J, Daponte A, Rodríguez M, Sánchez JJ. The influence of individual, social and physical environment factors on physical activity in the adult population in Andalusia, Spain. Int J Environ Res Public Health. 2010;7(1):60-77.

12. Brown $\mathrm{H}$, Roberts J. Exercising choice: the economic determinants of physical activity behaviour of an employed population. Soc Sci Med. 2011;73(3):383-90.

13. Hoehner CM, Ribeiro IC, Parra DC, Reis RS, Azevedo MR, Hino AA, et al. Physical activity interventions in Latin America: expanding and classifying the evidence. Am J Prev Med. 2013;44(3):e31-40.
14. Sallis JF, Cervero RB, Ascher W, Henderson KA, Kraft MK, Kerr J. An ecological approach to creating active living communities. Annu Rev Public Health. 2006;27:297-322.

15. Ferreira MS, Castiel LD, Cardoso MH. [Physical activity based on the new health promotion perspective: contradictions of an institutional program]. Cien Saude Colet. 2011;16(Suppl 1):865-72.

16. Carvalho T, Nóbrega ACL, Lazzoli JK, Magni JRT, Rezende L, Drummond FA, et al. Posição oficial da sociedade brasileira de medicina do esporte: atividade física e saúde. Rev Bras Med Esporte. 1999;(2)4:79-81

17. Hallal PC, Gomez LF, Parra DC, Lobelo F, Mosquera J, Florindo AA, Reis RS, Pratt M, Sarmiento OL. Lessons learned after 10 years of IPAQ use in Brazil and Colombia. J Phys Act Health. 2010;7(Suppl 2):S259-64.

18. Matsudo S, Araújo T, Matsudo V, Andrade D, Andrade E, Oliveira LC, et al. Questionário internaciona de atividade física (IPAQ): estudo de validade e reprodutibilidade no Brasil. Rev Bras Ativ Fis Saude. 2001;6(2):5-18

19. Craig CL, Marshall AL, Sjöström M, Bauman AE, Booth ML, Ainsworth BE, et al. International physica activity questionnaire: 12-country reliability and validity. Med Sci Sports Exerc. 2003;35(8):1381-95.

20. Ainsworth BE, Haskell WL, Herrmann SD, Meckes N, Bassett DR Jr, Tudor-Locke C, et al. 2011 Compendium of Physical Activities: a second update of codes and MET values. Med Sci Sports Exerc 2011;43(8):1575-81.

21. Brasil. Ministério da Saúde. Secretaria de Vigilância em Saúde. Vigitel Brasil 2011: Vigilância de Fatores de Risco e Proteção para Doenças Crônicas por Inquérito Telefônico. Ministério da Saúde, Secretaria de Vigilância em Saúde - Brasília: Ministério da Saúde; 2012. (Série G. Estatística e Informação em Saúde).

22. Florindo AA, Guimarães WV, Cesar CL, Barros MB, Alves MC, Goldbaum M. Epidemiology of leisure, transportation, occupational, and household physical activity: prevalence and associated factors. J Phys Act Health. 2009;6(5):625-32.

23. Del Duca GF, Nahas MV, Garcia LM, Mota J, Hallal PC, Peres MA. Prevalence and sociodemographic correlates of all domains of physical activity in Brazilian adults. Prev Med. 2013;56(2):99-102.

24. Rocha SV, Pie ACS, Cardoso JP, Amorim CR, Carneiro LRV, Vilela ABA. Nivel de atividade física entre funcionários de uma instituição de nível superior da Bahia. Ulbra e Movimento - Rev Educ Física. 2011;2(1):16-29.

25. Borsoi IC. Trabalho e produtivismo: saúde e modo de vida de docentes de instituições públicas de ensino superior. Cadernos de Psicologia Social do Trabalho. 2012;15 (1):81-100.

26. Bauman AE, Reis RS, Sallis JF, Wells JC, Loos RJ, Martin BW; Lancet Physical Activity Series Working Group. Correlates of physical activity: why are some people physically active and others not? Lancet. 2012;380(9838):258-71. 